\title{
A NEW CLASS OF HIGH FORCE, LOW-VOLTAGE, COMPLIANT ACTUATION SYSTEMS
}

\author{
M. Steven Rodgers', Sridhar Kota², Joel Hetrick ${ }^{3}$, Zhe Li², Brian D. Jensen², \\ Thomas W. Krygowski ${ }^{1}$, Samuel L. Miller ${ }^{1}$, Stephen M. Barnes ${ }^{1}$, and Michael S. Burg ${ }^{1}$ \\ 1) Intelligent Micromachine Department, Sandia National Laboratories \\ Albuquerque, New Mexico 87185-1080 \\ http://www.mems.sandia.gov
}

\author{
2) Department of Mechanical Engineering \\ and Applied Mechanics \\ The University of Michigan-Ann Arbor \\ Ann Arbor, Michigan 48109-2125
}

3) Department of Mechanical Engineering

The University of Wisconsin-Madison

Madison, Wisconsin 53706

\section{ABSTRACT}

Although many actuators employing electrostatic comb drives [1] have been demonstrated in a laboratory environment, widespread acceptance in mass produced microelectromechanical systems (MEMS) may be limited due to issues associated with low drive force, large real estate demands, high operating voltages, and reliability concerns due to stiction. On the other hand, comb drives require very low drive currents, offer predictable response, and are highly compatible with the fabrication technology. To expand the application space and facilitate the widespread deployment of self-actuated MEMS, a new class of advanced actuation systems has been developed that maintains the highly desirable aspects of existing components, while significantly diminishing the issues that could impede large scale acceptance. In this paper we will present low-voltage electrostatic actuators that offer a dramatic increase in force over conventional comb drive designs. In addition, these actuators consume only a small fraction of the chip area previously used, yielding significant gains in power density. To increase the stroke length of these novel electrostatic actuators, we have developed highly efficient compliant stroke amplifiers [2]. The coupling of compact, high-force actuators with fully compliant displacement multipliers sets a new paradigm for highly integrated microelectromechanical systems.

\section{INTRODUCTION}

Electrostatic comb drives [1] are ideal candidates for microelectromechanical applications requiring high frequency, very low current, and well controlled force generation over several microns of displacement. However, conventional comb drives typically only produce a few $\mu \mathrm{N}$ 's of drive force, while often requiring non-resonant mode operating voltages on the order of 100 volts [3]. In addition, conventional comb drives consume significant real estate and can occupy more than a square millimeter of chip area, placing a fundamental limit on the size and complexity of advanced microelectromechanical systems. In response to this, a new class of advanced actuation systems has been developed. Actuators that provide more than 200 times the force per unit area of previous designs and multipliers with gains from 5 to 50 and efficiencies greater than $80 \%$ (force out $\mathrm{x}$ displacement out $>0.80$ $\mathrm{x}$ force in $\mathrm{x}$ displacement in) have been designed, fabricated, and successfully demonstrated. As the method for synthesizing the novel displacement multipliers has previously been reported [2], this paper focuses on the design of the electrostatic actuation component.

\section{COMB DRIVE STABILITY ISSUES}

The equation governing the drive force generated by an electrostatic comb drive [3] is given in equation 1 :

$$
F=\frac{n \varepsilon t}{g} V^{2}
$$

Where $n=$ number of fingers, $\varepsilon=$ electrical permitivity, $t=$ finger thickness, $g=$ gap between fingers, and $V=$ voltage applied. Note that $n, t$, and $g$ are the parameters related to the mechanical design, with the goal being to maximize $F$ while consuming the least amount of die area. Therefore, $n$ and $t$ should be as large as possible and $g$ should be as small as possible. All of the devices presented in this paper were fabricated in the SUMMiT-V surface micromaching process $[4,5]$ at Sandia's Microelectronics Development Laboratory, so $t$ was maximized by always using every mechanical layer available in this technology to define the comb fingers. This leaves only $n$ and $g$ to work with. The highest finger density can be achieved by using the minimum width and space geometry permitted by the design rules, which are both 1.0 $\mu \mathrm{m}$ for this process. Although it is easy to design such a structure, to actually fabricate it and have it work is a different matter. This is because lateral instabilities become increasingly difficult to overcome as the comb geometry is downsized.

In addition to the force along the desired line of motion stated by equation 1 , there is a substantial parallel plate attractive force between each of the fingers as shown in Figure 1. Equation 2 shows this force is directly proportional to the area $A$ between the fingers and inversely proportional to the gap squared [3]. As long as the

$$
F_{p p}=\frac{\varepsilon A}{2 g^{2}} V^{2}
$$

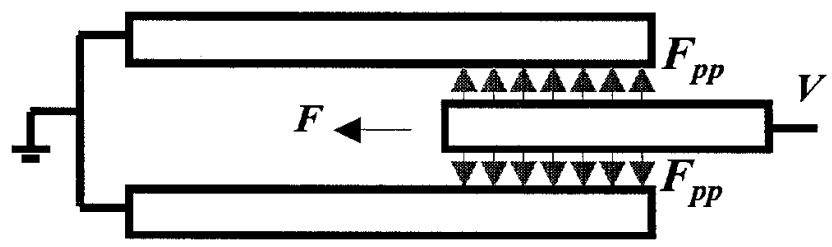

Figure 1. Forces acting on a comb drive finger when a voltage is applied. Vertical arrows represent parallel plate attraction. 


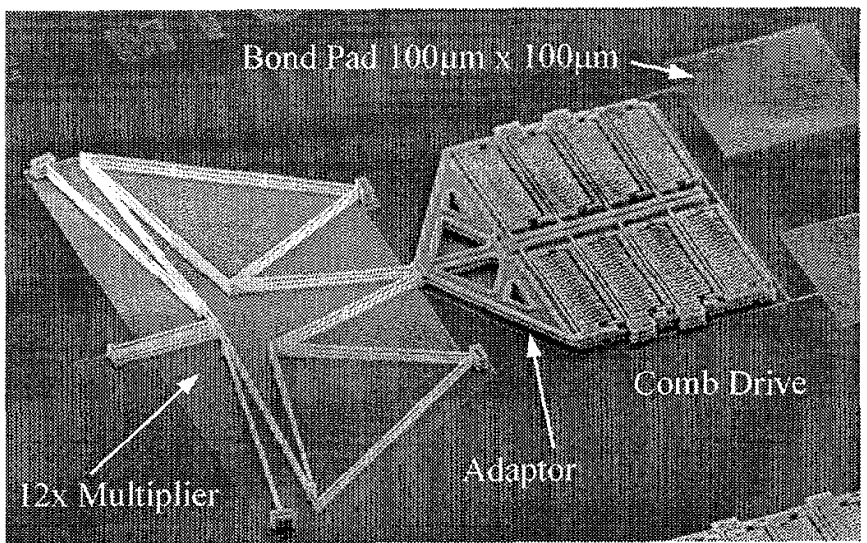

Figure 2. Scanning electron microscope (SEM) image of new comb drive with compliant displacement multiplier.

center comb finger rcmains precisely between the outer two, the parallel plate forces cancel. Any asymmetry or off axis loading, however, will create an imbalance that could easily overcome the restoring force of the structure resulting in lateral clamping of the comb fingers [6] and actuator failure. The issue with reducing the gaps between fingers to generate more force is this instability increases as the square of the gap dimension while the desired force only increases in direct proportionality. The problem is further compounded when very thin comb fingers are used to create a high density array, since their lateral stiffness varies as the cube of their width.

\section{DESIGNING FOR STABILITY}

The authors know from experience that developing a successful high force per unit area comb drive requires taking extreme care to ensure symmetry and mechanical stability. One of the actuation systems resulting from this effort is shown above in figure 2 . The first issue to be addressed was the prevention of lateral bending of thin highly-compliant comb fingers due to asymmetrical electrostatic fields. This is normally only a problem for the end fingers, since these typically see a comb finger on one side and very different geometry on the other. Therefore, the comb configuration in Figure 3 was employed to maintain balanced lateral forces on both sides of the fingers regardless of actuator displacement.

The second task was to create a rigid comb mounting structure that would not distort under high electrostatic forces. Common practice is to suspend banks of comb fingers by attaching one end to a single central beam and letting the other end float $[1,3]$. The new design incorporates outer beams as shown in Figure 4 to add additional support, significantly stiffening the assembly. The beams and the fingers mounted directly to them form the moving

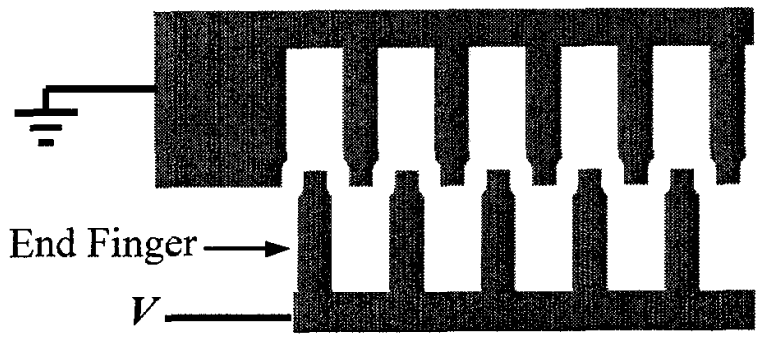

Figure 3. Balancing electrostatic forces on both sides of the thin comb fingers prevents shorting due to bending.

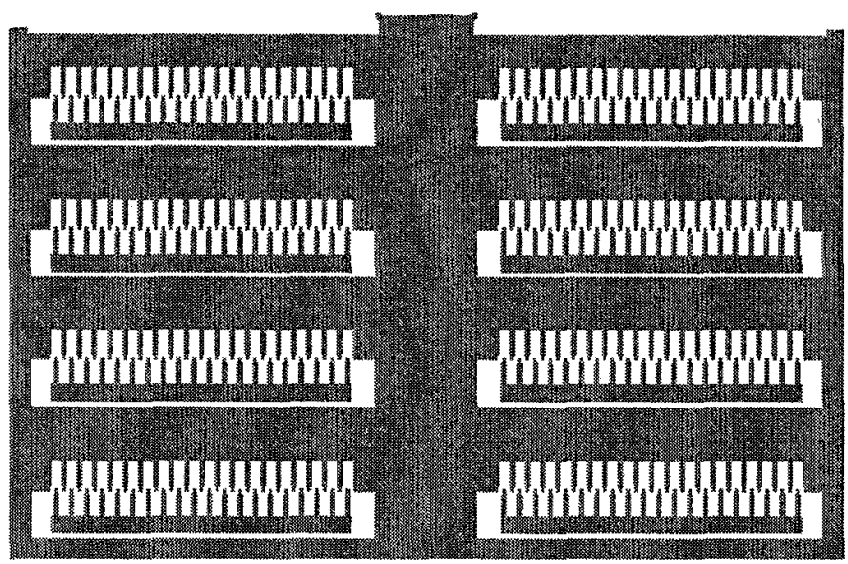

Figure 4. This structure firmly holds the banks of comb fingers in proper alignment under high electrostatic forces.

shuttle assembly, which is held at ground potential. Actuation occurs when voltage is applied to the stationary mechanically isolated banks of fingers. Desired motion is displacement of the shuttle towards the bottom of the figure. However, this movement is opposed by a parallel plate attraction force that develops between the main support beams for the stationary fingers and the nearby horizontal shuttle beams. This force is effectively eliminated by shiclding with grounded clectrostatic fences as shown in figure 5 .

Comb drive shuttle assemblies are supported by springs that must be compliant enough to allow movement along a line parallel to the fingers, yet stiff enough to prevent transverse movement which could result in lateral clamping of the comb fingers. These springs are normally attached to both sides of the shuttle assembly. Since this approach consumes significant amounts of valuable die area, a new high-stability suspension system was developed that allows the springs to be defined almost completely within the boundary established by the perimeter of the shuttle assembly. Support springs were fabricated in both the upper and lower mechanical layers and anchored together through openings cut in the central shuttle heam, which significantly increases the vertical or out of plane stiffness. The close-up in Figure 6 shows details of the suspension system and other previously described features.

The SUMMiT-V process has 4 mechanical levels of polysilicon and a poly0 electrical interconnect layer that also serves as a ground plane. The first 2 mechanical layers, poly1 and poly2, were laminated together to form a rigid $2.5-\mu \mathrm{m}$ thick lower layer that was patterned by a single mask, while the $2.25-\mu \mathrm{m}$ thick poly 3 and poly4 layers were used to form the middle and upper levels of the actuator. Therefore, only 3 mechanical levels are apparent.

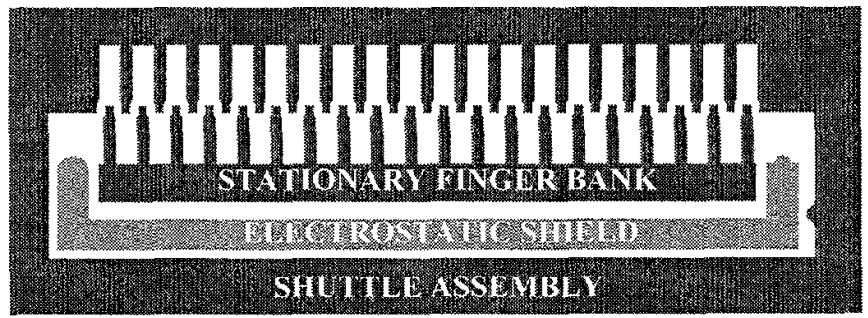

Figure 5. Grounded electrostatic shields prevent parallel plate attraction between the shuttle and stationary fingers. 


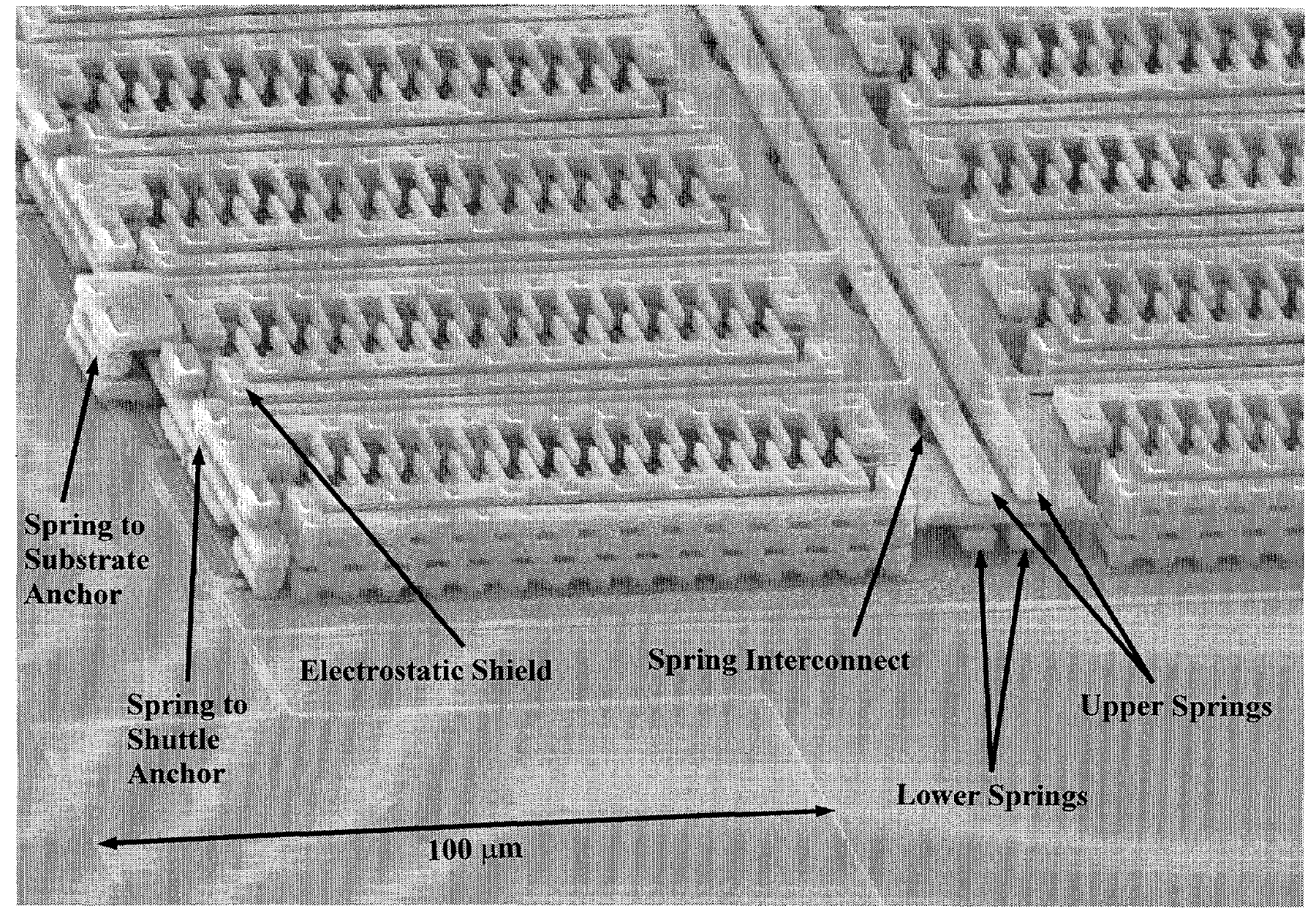

Figure 6. This SEM close-up of a portion of the new actuator assembly shows details of features discussed in text.

The actuator just described provides a $6-\mu \mathrm{m}$ stroke with full displacement being obtainable with just 22-35 volts, depending on the specifics of the suspension system. The actuator was also designed as a cluster of modular subassemblies that can be easily arrayed to provide the force necessary for the intended application. The largest fabricated to date is $1450-\mu \mathrm{m}$ long by $475-\mu \mathrm{m}$ wide, and has a calculated drive force of more than $2.5 \mathrm{mN}$ at 100 volts.

\section{SECOND ACTUATOR DESIGN}

A second actuator has been developed that only provides a $2-\mu \mathrm{m}$ stroke. However, it does this in a very small area. The entire electrode and shuttle assembly is just $116 \times 71 \mu \mathrm{m}$, or less than the area of a typical $100 \times 100-\mu \mathrm{m}$ bond pad. Yet it produces about the same force $(\sim 15 \mu \mathrm{N}$ at $100 \mathrm{~V})$ as the large $1200 \times 1000$ $\mu \mathrm{m}$ comb drives that Sandia has been using to drive the microengines [7]. Illustrated in Figure 7, this device has alternating rows of stationary electrodes that are anchored to the substrate, and electrodes that are connected to support beams. The beams are attached at both ends to a rigid rectangular frame which is held at ground potential. When a voltage is applied to the stationary fingers, the shuttle moves to align the electrodes horizontally as show in upper right of figure. This actuator features self-limiting displacement, so no mechanical stops are required. Once enough voltage is applied to almost fully displace the shuttle assembly, additional voltage provides very little additional displacement.

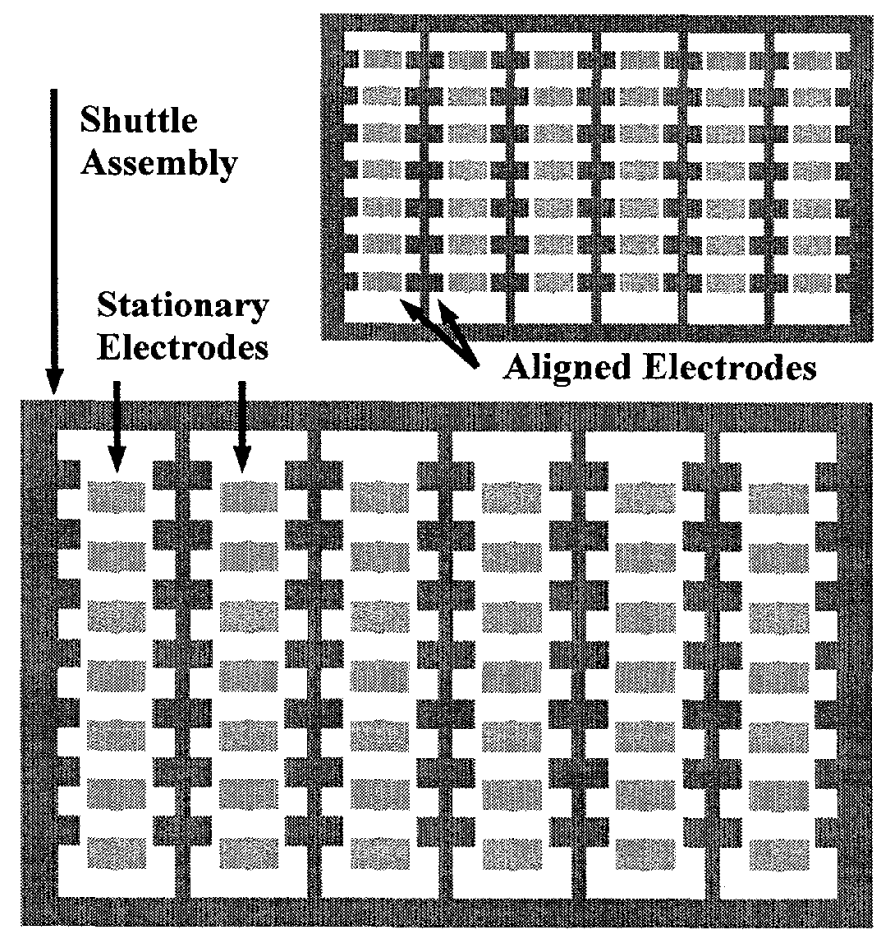

Figure 7. Second actuator design. Fabricated position shown on bottom; actuated position upper right. 


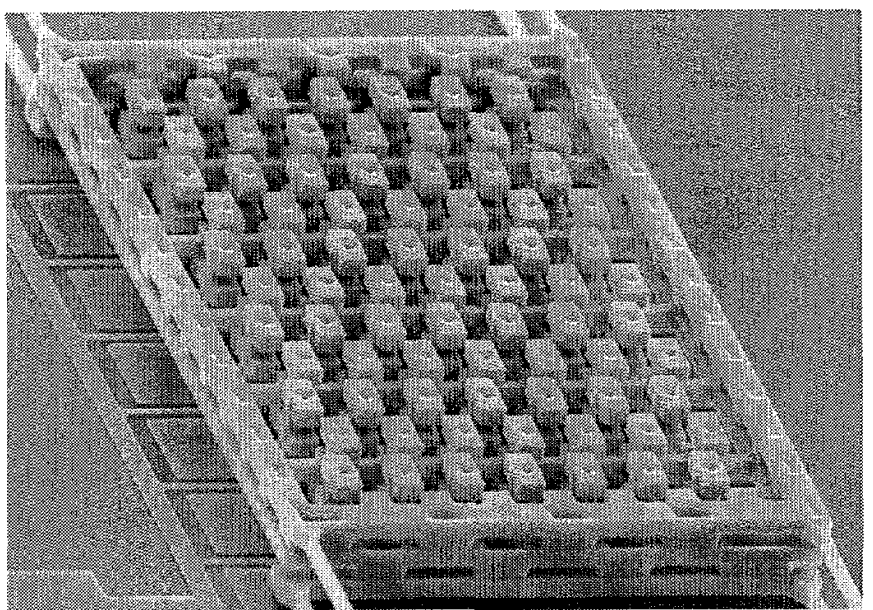

Figure 8. SEM image of second actuator design.

A close-up view of this actuator is shown above in Figure 8 . One benefit from designing this device to only move 2 or $3 \mu \mathrm{m}$ is that an extremely rigid and stable suspension system can be used. Simple $100-\mu \mathrm{m}$ to $200-\mu \mathrm{m}$ long and $2-\mu \mathrm{m}$ wide cantilever beams have successfully been used to support the structure shown in the figure. This device has demonstrated the ability to handle $190 \mathrm{~V}$, our test system limit, without becoming unstable. So very high forces can be generated in very small spaces in applications where supplying high voltage is not a problem.

Most applications, however, require lower voltage actuation. Like the actuator shown in figure 6 , thcse components were also designed to be modular. In figure 9 a $3 \times 3$ array of the actuator shown in figure 8 is being used in conjunction with a compliant displacement multiplier to create an actuator that provides enough displacement $(\sim 10 \mu \mathrm{m})$ at $30 \mathrm{~V}$ to drive a ratcheting component.

\section{CONCLUSIONS}

In spite of their generally high voltage and large die area requirements, surface micromachined electrostatic actuation systems have typically only provided marginal drive forces. This paper

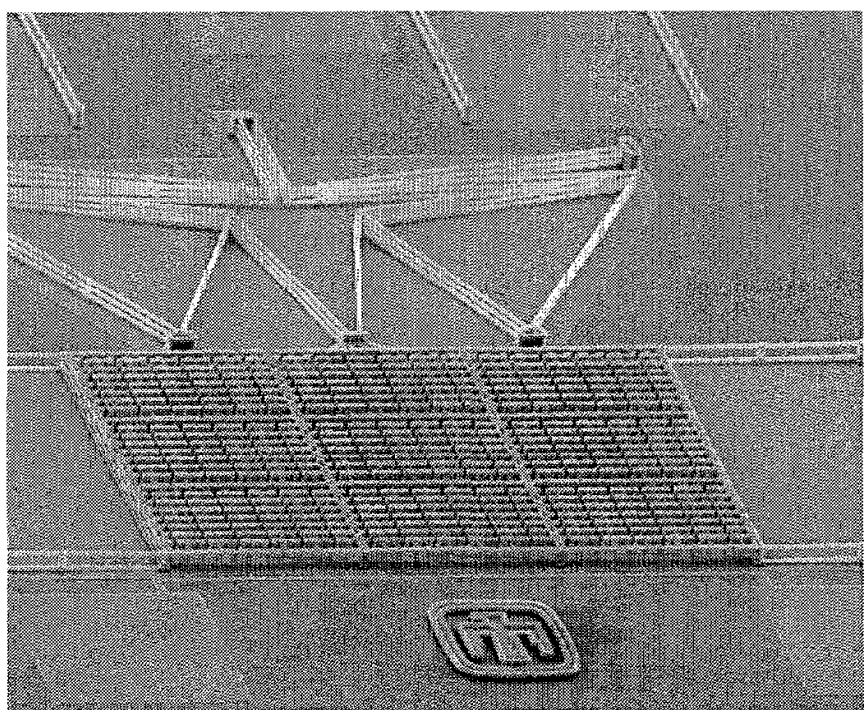

Figure 9. This compliant displacement multiplier is being driven with a $3 \times 3$ arroy of the actuator in figure 8 to reduce the voltage required for operation. shows that such attributes are not fundamental limitations. Novel short-stroke electrostatic actuators that provide dramatic increases in power density have becn demonstrated, and further gains are still possible. These devices can either be used directly or coupled to very efficient displacement multipliers to meet application requirements. Since drive force will be reduced by the multiplication factor, best performance will be obtained when the multiplier is optimized for a given actuator and load.

Yield and reliability are also improved compared to previous designs. The short, rigid suspension systems used in both the drives and multipliers keeps real estate requirements low while simultaneously making the structure very resistant to surface tension effects during the final release process, thus reducing stiction concerns.

The actuation systems described here are already being used in many of the surface micromachining programs at Sandia National Laboratories, and design enhancement is ongoing.

\section{ACKNOWLEDGEMENTS}

The authors are grateful to the personnel of the Microelectronics Development Laboratory at Sandia National Laboratories for fabricating the devices, to Gary Zender for the excellent SEM images, and to Jeff Sniegowski for developing the fabrication technology.

Sandia is a multiprogram laboratory operated by Sandia Corporation, a Lockheed Martin Company, for the United States Department of Energy under Contract DE-AC04-94AL85000.

\section{REFERENCES}

1) W. C. Tang, Ph.D. Thesis, University of California, Berkeley, CA. (1990).

2) Sridhar Kota, Joel Hetrick, Zhe Li, Steve Rodgers, and Thomas Krygowski, "Synthesing High-Performance Compliant Stroke Amplification Systems for MEMS", Proceedings of the IEEE Thirteenth International Micro Electro Mechanical Systems Conference, Miyazaki, Japan, 1/23-27/2000, pp. 164-169.

3) S. L. Miller, J. J. Sniegowski, G. LaVigne, and P. J. McWhorter, "Friction in surface micromachined microengines", Proc. SPIE Smart Electronics and MEMS, 2722, 2/28-29/96, San Diego, CA, (1996) pp. 197-204.

4) M. S. Rodgers and J. J. Sniegowski, "5-Level Polysilicon Surface Micromachine Technology: Application To Complex Mechanical Systems", Technical Digest of the 1998 Solid State Sensor and Actuator Workshop, Hilton Head Island, SC, 6/8-11/1998, pp. 144-149.

5) More technical information can be found at the web site http://www.mems.sandia.gov.

6) S. L. Miller, M. S. Rodgers, G. La Vigne, J. J. sniegowski, P. Clews, D. M. Tanner, and K. A. Peterson, "Failure Modes in Surface Micromachined Microelectromechanical Actuation Systems", Microelectronics Reliability, 39, 1229 (1999).

7) J. J. Sniegowski, S. L. Miller, G. LaVigne, M. S. Rodgers, and P. J. McWhorter, "Monolithic Geared Mechanisms Driven by a Polysilicon Surface Machined On-chip Electrostatic Engine", Technical Digest of the 1996 Solid State Sensor and Actuator Workshop, Hilton Head Island, SC, 6/3-6/96, Transducers Research Foundation, Cleveland (1996) pp.178-182. 